\title{
MECHANOCHEMICAL MODELS FOR GENERATING BIOLOGICAL PATTERN AND FORM IN DEVELOPMENT
}

\author{
J.D. MURRAY* and P.K. MAINI** \\ Centre for Mathematical Biology, Mathematical Institute, University of Oxford, UK \\ and \\ R.T. TRANQUILLO \\ Chemical Engineering and Materials Science, University of Minnesota, USA
}

Contents:

1. Introduction and background biology

2. Mechanical models for mesenchymal morphogenesis

61 4. Periodic patterns of feather germs 71

2.1. Cell equation

2.2. Cell-matrix mechanical interaction equation

2.3. Matrix conservation equation

625 5. Cartilage condensations in limb morphogenesis

63 6. A mechanochemical model for wound healing 76

65 7. Other applications of mechanochemical models 82

67 8. Discussion 82

3. Simple model: linear analysis, dispersion relation and pattern formation potential

Abstract:

The central issue in development is the formation of spatial patterns of cells in the early embryo. The mechanisms which generate these patterns are unknown. Here we describe the new Oster-Murray mechanochemical approach to the problem, the elements of which are experimentally well documented. By way of illustration we derive one of the basic models from first principles and apply it to a variety of problems of current interest and research. We specifically discuss the formation of skin organ patterns, such as feather and scale germs, cartilage condensations in the developing vertebrate limb and finally wound healing.

${ }^{*}$ Current address: Applied Mathematics, University of Washington, Seattle, WA, USA.

** Current address: Mathematics Department, University of Utah, Salt Lake City, UT, USA.

Single orders for this issue

PHYSICS REPORTS (Review Section of Physics Letters) 171, No. 2 (1988) 59-84.

Copies of this issue may be obtained at the price given below. All orders should be sent directly to the Publisher. Orders must be accompanied by check.

Single issue price Dfl. 19.00 , postage included. 


\title{
MECHANOCHEMICAL MODELS FOR GENERATING BIOLOGICAL PATTERN AND FORM IN DEVELOPMENT
}

\author{
J.D. MURRAY and P.K. MAINI
}

Centre for Mathematical Biology, Mathematical Institute, University of Oxford, UK and

\section{R.T. TRANQUILLO}

Chemical Engineering and Materials Science, University of Minnesota, USA

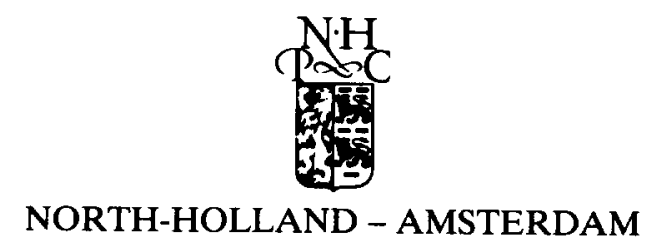




\section{Introduction and background biology}

Development of spatial pattern and form is one of the central issues in embryology and is included under the general name of morphogenesis. Pattern generation models are grouped together as morphogenetic models. These models provide the embryologist with possible scenarios as to how, and often when, pattern is laid down and how the embryonic form might be created. Although genes control pattern formation genetics says nothing about the actual mechanisms involved nor how the vast range of pattern and form that we see evolves from a homogeneous mass of dividing cells.

Broadly speaking the two prevailing views of pattern generation that have dominated the thinking of embryologists in the past few years are the long standing Turing (1952) chemical pre-pattern approach and the more recent mechanochemical approach developed by G.F. Oster and J.D. Murray and their colleagues [for example, Murray et al. 1983, Oster et al. 1983, Murray and Oster 1984a, b, Oster et al. 1985]. General descriptions have been given by Murray and Maini [1986] and Oster and Murray [1988].

Turing's theory [1952] of morphogenesis involves hypothetical chemicals, morphogens, which react and diffuse in such a way that if the kinetics have certain properties and the diffusion coefficients of the chemicals are not all the same then steady state heterogeneous spatial patterns in chemical concentrations can evolve from initial random perturbations about homogeneous steady states. Morphogenesis then proceeds by the cells reacting to the chemical prepattern and differentiating according to some bauplan [Wolpert 1969, 1981]. Turing's theory has stimulated a large amount of research, both theoretical and experimental. Such reaction diffusion models have been widely studied and applied to a variety of biological problems: see, for example [Murray 1977, 1981, Meinhardt 1982] and the book by Murray [1988]. The books by Fife [1979], Smoller [1983] and Britton [1986] give mathematical treatments of reaction diffusion systems. The dramatic Belousov-Zhabotinskii reaction is the best known reaction which will generate steady state concentration patterns. The book of articles edited by Field and Burger [1985] gives a good overview of this fascinating reaction.

In this paper we shall describe the comparatively new Oster-Murray mechanochemical approach, which directly brings forces and known properties of biological tissue into the process of morphogenetic pattern formation, and apply it to several specific developmental problems of current widespread interest in embryology.

The two approaches are basically quite different. In the chemical prepattern approach, pattern formation and morphogenesis takes place sequentially. First the chemical concentration pattern is formed, then the cells "read out" this pattern and the various cell differentiations, cell movement, and so on, follow from the chemical blueprint. So, in this approach morphogenesis is essentially a slave process which is determined once the chemical pattern has been established. Mechanical shaping of form which occurs during embryogenesis is not addressed in the chemical theory of morphogenesis. The continuing elusiveness of the chemical morphogens is also proving a considerable drawback in the acceptance of such a theory of morphogenesis. There is, however, no question but that chemicals play important roles in embryogenesis.

In the mechanochemical approach, pattern formation and morphogenesis is considered to go on simultaneously as a single process. An important aspect of this approach is that the models associated with it are formulated in terms of measurable quantities such as cell densities, forces, tissue deformation and so on. This focuses attention on the morphogenetic process itself and is more amenable to experimental investigation. The principal use of any theory is in its predictions and, even though each theory might be able to create similar patterns, they are mainly distinguished by the different experiments they suggest. 
A telling point in favour of simultaneous development is that such mechanisms have the potential for self-correction. Embryonic development is usually a very stable process with the embryo capable of adjusting to many outside disturbances. The process whereby a prepattern exists and then morphogenesis takes place is effectively an open loop system. These are potentially unstable processes and make it difficult for the embryo to make the necessary corrective adjustments as development proceeds.

Here we shall discuss morphogenetic processes which involve coordinated movement or patterning of populations of cells. The two types of early embryonic cells we shall be concerned with are fibroblast, or dermal or mesenchymal, cells and epidermal, or epithelial, cells. Fibroblast cells are capable of independent movement, due to long finger-like protrusions called filopodia or lamellapodia which grab onto adhesive sites on a tissue or external substratum and pull themselves along: spatial aggregation patterns in these appear as spatial variations in cell number density. Epidermal cells, on the other hand, in general do not move but are packed together in sheets and spatial patterns in their population are manifested by cell deformations. A notable exception is in the process of wound healing which is one of the applications we discuss in Section 6.

We first consider mesenchymal (fibroblast) cell pattern formation in early embryogenesis. In animal development the basic body plan is more or less laid down in the first few weeks. It is during this crucial early period that we expect pattern and form generating mechanisms, such as we propose here, to be operative. All of the models we describe are firmly based on macroscopic experimentally measurable variables and on generally accepted properties of embryonic cells.

In the following section we show how the models are derived and how a variety of effects can be incorporated if indicated from subsequent experiments. We should perhaps add here that these models pose numerous unsolved mathematical, both analytical and numerical, and biological modelling problems.

\section{Mechanical models for mesenchymal morphogenesis}

Several factors affect the movement of embryonic cells within a tissue substratum which is made up of extracellular matrix (ECM). For example, (i) convection, where cells may move passively on the ECM, due to the forces exerted on the ECM by other cells; (ii) contact guidance, in which the substratum on which cells crawl suggests a preferred direction; (iii) contact inhibition by cells, whereby a high density of neighbouring cells inhibits motion; (iv) haptotaxis, which we describe below, where the cells move up an adhesive gradient; (v) diffusion, where the cells move randomly but generally down a cell density gradient; (vi) galvanotaxis, where movement from the field generated by electric potentials, which are known to exist in embryos, provides a preferred direction of motion; (vii) chemotaxis, whereby a chemical gradient can direct cell motion both up and down a concentration gradient. These effects are all well documented from experiment.

The model field equations encapsulate the key features which affect cell movement within its extracellular environment. We shall not include all of the effects just mentioned but it will be clear how they can be incorporated and their effect quantified. The subsequent analysis of the field equations will show how regular patterned aggregates of cells come about. In sections 4 and 5 we shall describe two real applications of the model, namely to the highly organised patterns on skin such as the primordia which become feathers and scales, and the condensation of cells which presage the cartilage pattern in developing limbs. 
The basic mechanical model hinges on two key experimentally determined properties of mesenchymal cells in vitro: (i) cells migrate within a tissue substratum made up of fibrous extracellular matrix, the ECM, and other cells; (ii) cells can generate large traction forces [Harris et al. 1981]. The basic mechanism models the mechanical interaction between the motile cells and the viscoelastic substratum, within which they move.

Mesenchymal cells move by exerting forces on their surroundings, consisting of the viscoelastic fibrous ECM and the surface of other cells. They use their cellular protrusions, the filopodia or lamellapodia, which stretch out from the cell in all directions, to grip whatever is available and pull. As the cells move through the ECM they deform it by virtue of their traction forces. These deformations in the ECM induce anisotropy effects which in turn affect the cell motion. Analysis of models incorporating these various effects show that coordination of such effects result in spatially organised cell aggregations. The basic model is essentially that proposed by Murray et al. [1983], Murray and Oster [1984a, b], with a detailed biological description by Oster et al. [1983].

The model, a continuum one, consists of three equations governing (i) the equation for the cell population density, (ii) the mechanical balance of the forces between the cells and the ECM and (iii) the conservation law governing the ECM. Let $n(r, t)$ and $\rho(r, t)$ denote respectively the cell density (the number of cells per unit volume) and ECM density at position $r$ and time $t$. Denote by $u(r, t)$ the displacement vector of the ECM, that is, a material point in the matrix initially at position $r$ undergoes a displacement to $\boldsymbol{r}+\boldsymbol{u}$. We derive forms for each of these equations in turn.

\subsection{Cell equation}

This takes the general form

$$
\partial n / \partial t=-\nabla \cdot J+M
$$

where $J$ is the flux of cells, that is, the number crossing a unit area in unit time, and $M$ is the mitotic or cell proliferation rate. For simplicity we shall take a logistic form for the cell growth, namely $r n(N-n)$ where $r$ is the linear proliferative rate and $N$ is the maximum cell density in the absence of any other effects. We include in $\boldsymbol{J}$ some of the factors mentioned above which affect cell motion.

Convection. With $u(r, t)$ the displacement vector of the ECM the convective flux contribution $J_{\mathrm{c}}$ is

$$
J_{\mathrm{c}}=n \partial \boldsymbol{u} / \partial t
$$

Here the velocity of deformation of the matrix is $\partial u / \partial t$ and the amount of cells transported is simply $n$ times this velocity.

Random dispersal. Cells tend to disperse randomly when in a homogeneous isotropic medium. Classical diffusion contributes a flux term $-D_{1} \nabla n$, which models the random motion in which the cells respond to local variations in the cell density and tend to move down the density gradient. This results in the usual diffusion contribution $D_{1} \nabla^{2} n$ to the cell equation and represents local, or short range random motion.

In developing embryos the cell densities are relatively high and classical diffusion, which applies to dilute systems is not, perhaps, sufficiently accurate. The long filopodia extended by the cells can sense density variations beyond their nearest neighbours and so we must include a nonlocal effect on diffusive dispersal since the cells sense more distant densities. The appropriate form of the diffusion contribution 
can be derived from a Landau-Ginsberg approach [see Cohen and Murray 1981]. The flux of cells in this situation is modelled by

$$
J_{\mathrm{d}}=-D_{1} \nabla n+D_{2} \nabla\left(\nabla^{2} n\right)
$$

where $D_{1}>0$ is the usual Fickian diffusion coefficient and $D_{2}>0$ is the long range diffusion coefficient. The long range contribution gives rise to a biharmonic term in eq. (1) and has a stabilising effect if $D_{2}>0$. We can see this immediately if we consider the long range diffusion equation, obtained by substituting eq. (3) into eq. (1) and omitting the mitotic term $M$, to get

$$
\partial n / \partial t=-\nabla \cdot J_{\mathrm{d}}=D_{1} \nabla^{2} n-D_{2} \nabla^{4} n
$$

If we look for solutions of the form

$$
n(\boldsymbol{r}, t) \propto \exp [\boldsymbol{\sigma t}+\mathrm{i} \boldsymbol{k} \cdot \boldsymbol{r}]
$$

where $\boldsymbol{k}$ is the wavevector and substitute into the last equation we get the dispersion relation

$$
\sigma=-D_{2} k^{4}-D_{1} k^{2}<0
$$

for all wavenumbers $k(=|k|)$. So $n \rightarrow 0$ as $t \rightarrow \infty$ which implies $n=0$ is stable. If the biharmonic term had $D_{2}<0, n=0$ would be unstable for wavenumbers $k^{2}>-D_{1} / D_{2}$. To account for contact guidance, the diffusion coefficients may be functions of the strain tensor $\varepsilon\left(=\frac{1}{2}\left[\nabla u+\nabla u^{\mathbf{T}}\right]\right.$, where $\mathrm{T}$ denotes the transpose). Here however we shall take them to be constants.

Haptotaxis or Mechanotaxis. The traction exerted by the cells on the matrix generates gradients in the matrix density $\rho(r, t)$. We associate the density of matrix with the density of adhesive sites for the cell lamellapodia to get a hold of. Cells free to move in an adhesive gradient tend to move up it since they can get a stronger grip on the denser matrix. This results in a net flux of cells up the gradient which gives the haptotactic flux as

$$
J_{\mathrm{h}}=a n \nabla \rho
$$

where $a>0$.

The cell equation (1), with the flux contributions to $J$ from eqs. (2)-(4) and a logistic form for the mitosis $M$, becomes

$$
\begin{aligned}
& \partial n / \partial t=-\nabla \cdot(n \partial u / \partial t)+\nabla \cdot\left[D_{1} \nabla n-D_{2} \nabla\left(\nabla^{2} n\right)\right] \quad \text { (convection }+ \text { diffusion) } \\
& -\nabla \cdot(a n \nabla \rho)+r n(N-n) \quad \text { (haptotaxis + mitosis), }
\end{aligned}
$$

where $D_{1}, D_{2}, a, r$ and $N$ are positive parameters.

We have not included galvanotaxis and chemotaxis in eq. (5) but we can easily deduce what such a contribution would look like. For example, if $\phi$ is the electric potential then the galvanotactic flux can be written as

$$
J_{\mathrm{g}}=g n \nabla \phi
$$


where the parameter $g>0$. If cells responded to a chemoattractant $c$, chemotactic flux would be

$$
J_{\text {chem }}=n \chi \nabla c,
$$

where $\chi(c)$ is a function of the attractant. There are other refinements which could also be included; see for example [Murray 1988].

Although eq. (5) is clearly not the most general possible it suffices to show what can be expected in more realistic model mechanisms for biological pattern generation.

The analysis of such models allows us to compare the various effects as to their pattern formation potential and hence to come up with the simplest realistic system which can generate pattern and which is experimentally testable.

\subsection{Cell-matrix mechanical interaction equation}

The composition of the fibrous extracellular matrix, the ECM, within which the cells move is complex and moreover, its constituents change as development proceeds. Here we are interested in the mechanical interaction between the cells and the matrix. As the mechanical deformations are small a reasonable first approximation is to consider the composite material of cells plus matrix to be modelled as a linear, isotropic viscoelastic continuum with stress tensor $\boldsymbol{\sigma}(\boldsymbol{r}, t)$.

The time scale of embryonic motions during development is very long (hours) and the spatial scale is very small (less than a few millimetres). We can thus ignore inertial effects in the mechanical equation for the cell-ECM interaction (we are in a small Reynolds number regime) and take the traction forces generated by the cells to be in mechanical equilibrium with the viscoelastic restoring forces developed in the matrix and any external forces present. The mechanical cell-matrix equation is then [see, for example, Landau and Lifshitz 1970]

$$
\boldsymbol{\nabla} \cdot \boldsymbol{\sigma}+\rho \boldsymbol{F}=0
$$

where $\boldsymbol{F}$ is the external force (per unit matrix density) acting on the matrix and $\boldsymbol{\sigma}$ is the stress tensor. Consider first the stress tensor $\boldsymbol{\sigma}$. It consists of contributions from the ECM and the cells and we write

$$
\boldsymbol{\sigma}=\boldsymbol{\sigma}_{\mathrm{ECM}}+\boldsymbol{\sigma}_{\text {cell }}
$$

The usual expression for a linear viscoelastic material [Landau and Lifshitz 1970] gives the stress-strain constitutive relation as

$$
\boldsymbol{\sigma}_{\mathrm{ECM}}=\left[\mu_{1} \varepsilon_{t}+\mu_{2} \theta_{t} I\right]+E^{\prime}\left[\boldsymbol{\varepsilon}+\nu^{\prime} \theta I\right] \quad \text { (viscous }+ \text { elastic) }
$$

where

$$
E^{\prime}=E /(1+\nu), \quad \nu^{\prime}=\nu /(1-2 \nu)
$$

Here the subscript $t$ denotes partial differentiation with respect to time, $I$ is the unit tensor, $\mu_{1}, \mu_{2}$ are the shear and bulk viscosities of the ECM, $\varepsilon$ and $\theta$ are respectively the strain tensor and dilation defined 
by

$$
\varepsilon=\frac{1}{2}\left(\nabla u+\nabla u^{\mathrm{T}}\right), \quad \theta=\nabla \cdot u
$$

and $E$ and $\nu$ are the Young's modulus and Poisson ratio respectively.

Fibrous materials are also characterised by nonlocal elastic interactions since the fibres can transmit stress between points in the ECM quite far apart. By arguments analogous to those which lead to the biharmonic term in the cell equation (5) we should include long range effects in the elastic stress for the composite material. We could also introduce anisotropy by having a $\theta$-dependence for $E$ and $\nu$ but, again, do not do so here.

Now consider the contribution to the stress tensor from the cell tractions, that is $\boldsymbol{\sigma}_{\text {cell }}$. The more cells there are the greater the traction force. There is, however, experimental evidence indicating cell-cell contact inhibition with the traction force decreasing for large enough cell densities. This can be simply modelled by assuming that the cell traction forces $\tau(n)$ per unit mass of matrix initially increase with $n$ but eventually decrease with $n$ for large enough $n$. Here we simply choose

$$
\tau(n)=\tau n /\left(1+\lambda n^{2}\right),
$$

where $\tau$ [dyne $\mathrm{cm} / \mathrm{gm}$ ] is a measure of the traction force generated by a cell and $\lambda>0$. Typical experimental values for $\tau$ are of the order of $10^{-3}$ dyne $/ \mu \mathrm{m}$ of cell edge, which is a very substantial force [Harris et al. 1981].

As the filopodia, with which the cells attach to the ECM, extend beyond their immediate neighbourood, it is again reasonable to include a nonlocal effect analogous to the long range diffusion effect we included in the cell equation. We thus take the contribution $\sigma_{\text {cell }}$ to the stress tensor to be

$$
\sigma_{\text {cell }}=\tau\left(1+\lambda n^{2}\right)^{-1} n\left(\rho+\gamma \nabla^{2} \rho\right) I,
$$

where $\gamma>0$ is the measure of the nonlocal long-range cell-ECM interaction. The long range effects here are more important than the long range diffusion effect in the cell equation.

Finally consider the body force $\boldsymbol{F}$ in eq. (7). With the applications discussed below, the matrix material is attached to a substratum of underlying tissue by what can perhaps be best described as kinds of guy ropes. We model these restraining forces as body forces per unit matrix density proportional to the displacement of the matrix from its unstrained position and thus take

$$
\boldsymbol{F}=-s \boldsymbol{u},
$$

where $s>0$ is an elastic parameter characterising the substrate attachments.

Thus the force equation we take for the mechanical equilibrium between the cells and the ECM is eq. (7), with eqs. (8)-(14), which gives

$$
\begin{aligned}
& \nabla \cdot\left[\mu_{1} \varepsilon_{t}+\mu_{2} \theta_{t} I+E^{\prime}\left(\varepsilon+\nu^{\prime} \theta l\right)+\tau n\left(1+\lambda n^{2}\right)^{-1}\left(\rho+\gamma \nabla^{2} \rho\right) /\right]-s \rho \boldsymbol{u}=0, \\
& \text { (viscous + elastic + cell traction + external forces) }
\end{aligned}
$$

where $E^{\prime}$ and $\nu^{\prime}$ are defined by eq. (10). 


\subsection{Matrix conservation equation}

The conservation equation for the matrix material, $\rho(r, t)$, is

$$
\partial \rho / \partial t+\nabla \cdot(\rho \partial u / \partial t)=0
$$

that is, the matrix only moves by convection. At certain times the cells secrete matrix, but not with the applications we shall be discussing so we omit it here.

Equations (5), (15) and (16) constitute the field equations for our model pattern formation mechanism for fibroblast cells. The model involves 13 parameters, namely $D_{1}, D_{2}, a, r, N, \mu_{1}, \mu_{2}, \tau, \lambda$, $\gamma, s, E$ and $\nu$, all of which are in principle measurable and some of which are currently being investigated experimentally.

To assess the relative importance of the various effects we nondimensionalize the equations. We use general length and time scales $L$ and $T$, typical cell and matrix densities $N$ and $\rho_{0}$ and set

$$
\begin{aligned}
& r^{*}=r / L, \quad t^{*}=t / T, \quad n^{*}=n / N, \quad u^{*}=u / L, \quad \rho^{*}=\rho / \rho_{0}, \\
& \nabla^{*}=L \nabla, \quad \theta^{*}=\theta, \quad \boldsymbol{\varepsilon}^{*}=\varepsilon, \quad \gamma^{*}=\gamma / L^{2}, \quad r^{*}=r N T, \\
& s^{*}=s \rho_{0} L^{2}(1+\nu) / E, \quad \lambda^{*}=\lambda N^{2}, \quad \tau^{*}=\tau \rho_{0} N(1+\nu) / E \\
& a^{*}=a \rho_{0} T / L^{2}, \quad \mu_{i}^{*}=\mu_{i}(1+\nu) / T E, \quad i=1,2 \\
& D_{1}^{*}=D_{1} T / L^{2}, \quad D_{2}^{*}=D_{2} T / L^{4} .
\end{aligned}
$$

Depending on what time and length scales we are particularly concerned with we can reduce the parameter set further. For example, if we choose $T$ as the mitotic time $1 / r N$, then $r^{*}=1$ : this means we are interested in the evolution of pattern on the mitotic time scale. Alternatively we could choose $T$ so that $\gamma^{*}=1$ or $\mu_{1}=1$ for $i=1$ or 2 . Similarly we can choose a relevant length scale and further reduce the number of groupings.

The model mechanism is thus, on dropping the asterisks for notational simplicity,

$$
\begin{aligned}
& n_{t}=D_{1} \nabla^{2} n-D_{2} \nabla^{4} n-\nabla \cdot(a n \nabla \rho)-\nabla \cdot\left(n u_{t}\right)+r n(1-n), \\
& \nabla \cdot\left\{\left(\mu_{1} \varepsilon_{t}+\mu_{2} \theta_{t} I\right)+\left(\varepsilon+\nu^{\prime} \theta I\right)+\left[\tau n /\left(1+\lambda n^{2}\right)\right]\left(\rho+\gamma \nabla^{2} \rho\right) I\right\}=s \rho u, \\
& \rho_{t}+\nabla \cdot\left(\rho u_{t}\right)=0 .
\end{aligned}
$$

Although the model system is analytically formidable the model's conceptual framework is quite clear, as illustrated in fig. 2.1.

This model does not include all the effects that might be relevant, however it suffices to show how such realistic models can be constructed. One of the major roles of such modelling and subsequent analysis is to indicate just what features are essential for pattern formation. Although the following linear analysis can be carried out for the full system we simply want to demonstrate the pattern formation potential and so look at a simple version of the model (18-20) in which we set various parameters to zero. 


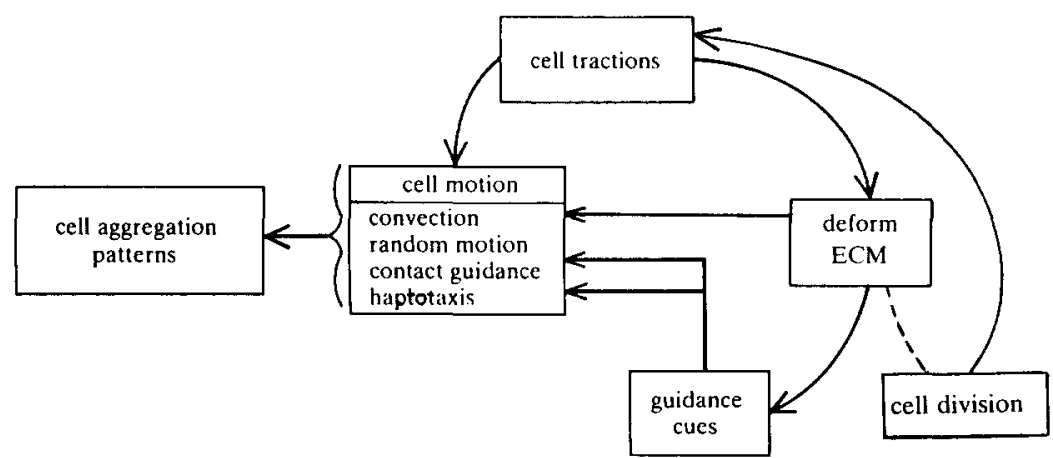

Fig. 2.1. Conceptual framework for the mechanical models. Cell tractions play a central role in orchestrating pattern formation.

\section{Simple model: linear analysis, dispersion relation and pattern formation potential}

To model spatial aspects observed in embryonic development the basic system (18-20) must admit spatially inhomogeneous solutions. There is little hope, at this stage, of finding useful analytic solutions to such nonlinear systems. However, much of the pattern formation potential is predicted by a linear analysis about uniform steady state solutions. Such predictions must be backed up by a nonlinear theory or by numerical simulations if finite amplitude structures far from homogeneity are required.

Let us consider a particularly simple version of (18-20) by taking diffusion and haptotaxis to be negligible, that is $D_{1}=D_{2}=a=0$. Further consider the situation with no cell proliferation so $r=0$ and where, for illustrative purposes only, there is no cell-cell inhibition so $\lambda=0$. In this situation the one-dimensional version of the model mechanism becomes

$$
\begin{aligned}
& n_{t}+\left(n u_{t}\right)_{x}=0 \\
& \mu u_{x x t}+u_{x x}+\left[\tau n\left(\rho+\gamma \rho_{x x}\right)\right]_{x}=s \rho u \\
& \rho_{t}+\left(\rho u_{t}\right)_{x}=0
\end{aligned}
$$

where $\mu=\mu_{1}+\mu_{2}$ and we have rescaled $\mu, \tau$ and $s$ by $E(1+\nu)$. The implication of the simple conservation equation for $n$ is that the cells are simply convected by the matrix. This is believed to be the major transport process.

The uniform steady state solutions of eq. (21) are

$$
n=u=\rho=0 ; n=1, \rho=0, u=1 ; n=\rho=1, u=0
$$

The first two solutions are not relevant as $\rho$ is zero. In fact there is a general steady state $n=n_{1}, \rho=\rho_{1}$, $u=0$ where $n_{1}$ and $\rho_{1}$ are constants. By suitable nondimensionalisation we can take these constants to be 1 . The linear stability of the third solution is found in the usual way by seeking solutions of (21) linearized about this steady state. We thus consider $n-1, \rho-1$ and $u$ to be small and on substituting into the nonlinear system and retaining only linear terms in $n-1, \rho-1$ and $u$ we get the following linear system, where for algebraic convenience we have written $n$ and $\rho$ for $n-1$ and $\rho-1$ respectively: 


$$
\begin{aligned}
& n_{t}+u_{x t}=0 \\
& \mu u_{x x t}+u_{x x}+\left(\tau n+\tau \rho+\tau \gamma \rho_{x x}\right)_{x}=s u \\
& \rho_{t}+u_{x t}=0
\end{aligned}
$$

Now look for solutions by setting

$$
(n, \rho, u) \propto \exp [\sigma t+\mathrm{i} k x]
$$

where $k$ is the wavenumber and $\sigma$ is the linear growth factor. Substitution of eq. (24) into (23) gives the dispersion relation $\sigma=\sigma\left(k^{2}\right)$ as

$$
\begin{aligned}
& \sigma\left(k^{2}\right)=-b\left(k^{2}\right) / 2 \mu k^{2}, \\
& b\left(k^{2}\right)=\gamma \tau k^{4}+(1-2 \tau) k^{2}+s .
\end{aligned}
$$

Spatially heterogeneous solutions of the linear system are characterised by a dispersion $\sigma\left(k^{2}\right)$ which has $\sigma(0) \leq 0$ but which exhibits a range of unstable modes with $\sigma\left(k^{2}\right)>0$ for $k^{2} \neq 0$. All the solutions (24) with these $k$ 's are then linearly unstable and grow exponentially with time. In the usual way we expect these unstable heterogeneous linear solutions to evolve into finite amplitude spatially structured solutions.

From eq. (25), if $k^{2}=0$, the spatially homogeneous case, $b(0)=s>0$ and so $\sigma\left(k^{2}\right)<0$ for small enough $k$. For $\sigma\left(k^{2}\right)>0$, to exist for at least some $k^{2} \neq 0$, requires from the expression for $b\left(k^{2}\right)$ in eq. (25), that for a range of $k^{2}, b\left(k^{2}\right)<0$; that is at least $\tau>1 / 2$. Since

$$
b_{\min }=s-(2 \tau-1)^{2} / 4 \gamma \tau,
$$

we thus have a range of $k^{2}>0$ where $\sigma\left(k^{2}\right)>0$ if

$$
\tau>\tau_{\mathrm{c}}=(1+\gamma s)+\left[(1+\gamma s)^{2}-1\right]^{1 / 2}
$$

This defines a bifurcation surface in $(\tau, \gamma, s)$ parameter space wherein the homogeneous steady state is unstable to spatial disturbances with wavenumbers

$$
k_{1}^{2}<k^{2}<k_{2}^{2}, \quad k_{1}^{2}, k_{2}^{2}=\left\{(2 \tau-1) \pm\left[(2 \tau-1)^{2}-4 s \gamma \tau\right]^{1 / 2}\right\} / 2 \gamma \tau,
$$

where $\tau, \gamma$ and $s$ must satisfy eq. (26). There is a fastest growing linear mode, the one with wavenumber giving $\sigma_{\max }$ which predicts, usually only in one-dimensional models with random initial conditions, the ultimate nonlinear spatial pattern.

From the dispersion relation (25), the only way a solution with $\sigma\left(k^{2}\right)>0$ can exist is if $b\left(k^{2}\right)<0$, which in turn relies on a large enough cell traction $\tau$, given by eq. (26), to be precise. It is also clear heuristically from the mechanism that this has to be the case since the cell traction forces are the only contribution to the aggregative process in the force-balance equation in eq. (21). Typically values for the cell traction, in vitro are of the order of $10^{-3} \mathrm{~N} \mathrm{~m}^{-1}$ of cell edge.

When eq. (26) is satisfied the dispersion relation (25) is a typical basic dispersion relation of the type which initiates spatial patterns: it is illustrated in fig. 3.1. 


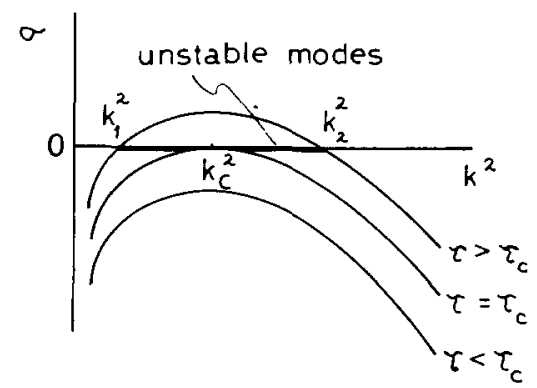

Fig. 3.1. Behaviour of the dispersion relation $\sigma\left(k^{2}\right)$, for the model (23). As the traction, $\tau$, increases above a critical $\tau_{\mathrm{c}}$, given by eq. (26), wavenumbers in the interval $\left[k_{1}^{2}, k_{2}^{2}\right]$, given by eq. (27), go unstable.

The system (18-20) can display an astonishing range of dispersion relations depending on the model parameters. These have been analysed by Murray and Oster [1984a]. Figure 3.2 is only a selection which demonstrates the richness of dispersion relation types wherein a range of wavenumbers are linearly unstable.

The nonlinear singular perturbation technique developed by Maini and Murray [1988] can be used on mechanisms with such dispersion relations of the type illustrated in fig. 3.1. They also considered two-dimensional mechanisms and obtained roll and hexagonal solutions. The significance of the latter will be discussed in the following section on a biological application to skin organ morphogenesis.

Perhaps it should be mentioned here that the spectrum of spatial patterns possible with the mechanism (18-20) and its numerous simplifications is orders of magnitude greater than with a reaction-diffusion system - even three-species systems. The implications of a paper by Penrose [1979] indicate that tensor systems have solutions with a wider class of singularities than vector systems. Since the cell-matrix equation is a tensor equation, its solutions should thus include a wider class of singularities than reaction-diffusion vector systems. Even with the linear systems analytical and numerical studies have only just started. Another property of models such as (21) is that the final

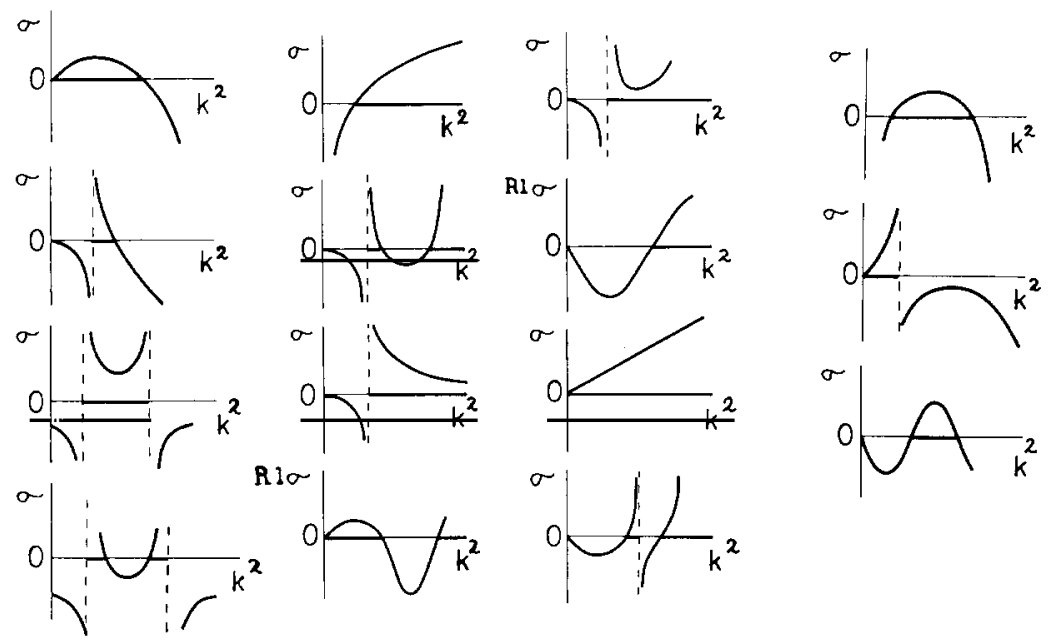

Fig. 3.2. Some examples of dispersion relations $\sigma\left(k^{2}\right)$, obtained from the mechanical model mechanism (18-20) on setting various parameters to zero. Realistic models for those with infinite linear growth must be treated as singular perturbation problems, with small values for the appropriate parameters in terms which have been omitted so as to make the linear growth finite although large. 
solution will depend on the initial conditions, that is, since cell density is conserved, different initial conditions (and hence different cell number) will give rise to different patterns. However, as the random perturbations are small, the differences in the final solution are also small. This seems biologically realistic as no two patterns are exactly the same.

In the following two sections we consider two biologically important and widely studied pattern formation problems using mechanical models of pattern generation.

\section{Periodic patterns of feather germs}

Generation of regular patterns occurs in many situations in early embryogenesis. These are particularly evident in skin organ morphogenesis such as in the formation of feather and scale primordia and are widely studied [see, for example, Sengel 1976, Davidson 1983a, b]. Feather formation has much in common with scale formation during the early developmental phase of primordia. Here we shall concentrate on feather germ formation with particular reference to the chick, and fowl in general. Feather primordial structures are distributed across the surface of the animal in a characteristic and regular hexagonal fashion. The application of the Oster-Murray mechanical theory to feather germ primordia was first put forward by Murray et al. [1983] and Oster et al. [1983] and it is their scenario we describe here. We first present the biological background which suggests a mechanical model.

Vertebrate skin consists essentially of two layers; an epithelial epidermis overlays a much thicker mesenchymal dermis and is separated from it by a fibrous basal lamina. In general, epithelial cells do not move, but the epithelial layer can deform through buckling. Dermal cells are loosely packed and motile and can move around in the extracellular matrix, the ECM, as we described earlier. The earliest observable developmental stages of feather and scale germs begin the same way. We shall concentrate here on the initiation and subsequent appearance of feather rudiments in the dorsal pteryla - the feather forming region on the chick back.

In the chick the first feather rudiments become visible about 6 days after egg fertilization. Each feather germ, or primordium, consists of a thickening of the epidermis with one or more layers of columnar cells, called a placode, beneath which is an aggregation of dermal (mesenchymal) cells, called a papilla. The dermal condensations are largely the result of cell migration, with localised proliferation playing a secondary role. Whether or not the placodes form prior to the dermal papillae is a controversial issue. There is considerable experimental work going on to determine the order of appearance or, indeed, whether the epidermis and dermis interact to produce their patterns simultaneously. The dermis seems to determine the spatial patterning-as shown by epidermal-dermal recombination experiments [Rawles 1963, Dhouailly 1975]. The model we discuss here is for the formation of dermal papillae. Subsequent development, however, is a coordinated process involving both the epidermal and dermal layers [Sengel 1976, Wessells 1977].

Davidson [1983a, b] demonstrates that chick feather primordia appear sequentially. A central column of dermal cells forms on the dorsal pteryla and subsequently breaks up into a row of papillae. As the papillae form, tension lines develop joining the cell aggregation centres. With the above mechanical models this is consistent with the cells trying to align the ECM. Now lateral rows of papillae form sequentially but these are interdigitated with the papillae in the preceding row: see fig. 4.1. These lateral rows spread out from the central midline almost like a wave of pattern initiation. Experiments by Davidson [1983] tend to confirm this wave theory - later we show how these results can be explained by our model and we present corroborative numerical results. 


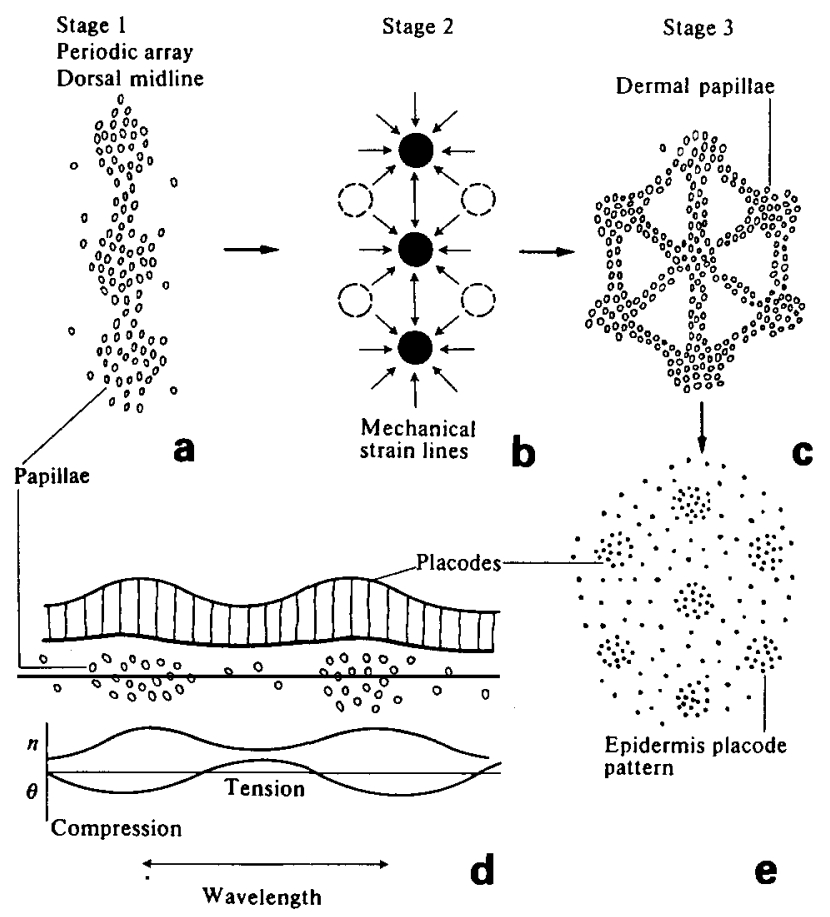

Fig. 4.1. (a) This shows the predicted breakup of a uniform distribution of mesenchymal (dermal) motile cells into regular cell condensations with a wavelength determined by the parameters of the model mechanism (stage 1). These cell aggregations are the primordial papillae for feathers and scales. (d) Vertical cross section qualitatively showing the feather germ primordia. The placodes in the epidermis are underlain by the papillae which create the stress field. (b) Subsequent aggregations form laterally. The prestressed strain field from the first line of condensations induces a bias so that the neighbouring line of papillae interdigitate with the first line (stage 2). The resulting periodic array is thus hexagonal, the basic unit of which is illustrated in (c) (stage 3). (e) The epidermal placode pattern that mirrors the dermal pattern.

These observations suggest that it is reasonable first to model the pattern formation process for the initial row of papillae by a one-dimensional column of cells and look for the conditions for spatial instability which generates a row of papillae. This is stage 1 and is illustrated in fig. 4.1a.

We have seen in the previous sections that even the simple mechanical model (21) can generate spatial patterns as the cell traction parameter $\tau$ increases beyond some critical value $\tau_{\mathrm{c}}$. At this value a mode with a specific wavenumber $k_{\mathrm{c}}$, that is, with wavelength $2 \pi / k_{\mathrm{c}}$, first becomes unstable and a spatial pattern starts to evolve: this generates a regular pattern of dermal papillae.

Simulations of the one-dimensional version of the following nonlinear mechanical model, a simpler version of the more comprehensive model (18-20), have been carried out by Perelson et al. [1986], viz,

$$
\begin{aligned}
& \partial n / \partial t=D_{1} \partial^{2} n / \partial x^{2}-\partial / \partial x(n \partial u / \partial t)-a \partial / \partial x(n \partial \rho / \partial x), \\
& \partial / \partial x\left\{\mu \partial^{2} u / \partial x \partial t+\partial u / \partial x+\left[\tau n /\left(1+\lambda n^{2}\right)\right]\left(\rho+\gamma \partial^{2} \rho / \partial x^{2}\right)\right\}=s u \rho, \\
& \partial \rho / \partial t+\partial / \partial x(\rho \partial u / \partial t)=0 .
\end{aligned}
$$

They particularly addressed the problem of mode selection in models with many parameters, and proposed a simple scheme for determining parameter sets to isolate and "grow" a specific wavelength 
pattern. Figure 4.2a shows a typical steady state pattern of cell aggregations (the papillae), ECM density variations and matrix displacement. As we would expect intuitively, the cell aggregations are in phase with the ECM density variations $\rho$, and both are out of phase with the ECM displacement $u$. The reason is that the cell aggregations pull the matrix towards the areas of higher cell density thus stretching the matrix between them: fig. $4.1 \mathrm{~d}$ illustrates what is going on physically.

Patterns of the type illustrated in fig. 4.1a occur only if the cell traction parameter is above a certain critical value (see section 3). Thus a possible scenario for the formation of the pattern along the dorsal midline is that there is a wave of initiation that sweeps down the column which could be related to tissue age; in this case it is known (from in vitro experiments) that the cell traction parameter increases. As the cells become stronger $\tau$ passes through the critical value $\tau_{\mathrm{c}}$ and pattern in initiated.

Let us now consider the formation of the distinctive hexagonal two-dimensional pattern of papillae. We described above how a wave of pattern initiation seems to spread out from the dorsal midline. This means that the pattern of matrix strains set up by the initial row of papillae biases the formation of the secondary condensations at positions displaced from the first line by half a wavelength. Figure $4.2 \mathrm{~b}$ shows the resulting numerical simulation: note how the patterns are out of phase with those in fig. 4.2a. If we now look at figs. $4.1 \mathrm{~b}, \mathrm{c}$ we see how this scenario generates a regular hexagonal pattern in a sequential way like a wave emanating from the central dorsal midline.

This "wave" is, however, not a wave in the usual sense since if the dermal layer is cut along a line parallel to the dorsal midline the wave simply starts up again beyond the cut ab initio. This is consistent with Davidson's experimental observations [1983b].

The modelling here does not cast light on the controversy regarding the order of formation of placodes and papillae. However, since the traction forces generated by the dermal cells can be quite large the model lends support to the view that the dermis controls the pattern even if it does not initiate it. Current thinking tends towards the view that initiation requires tissue interaction between the dermis
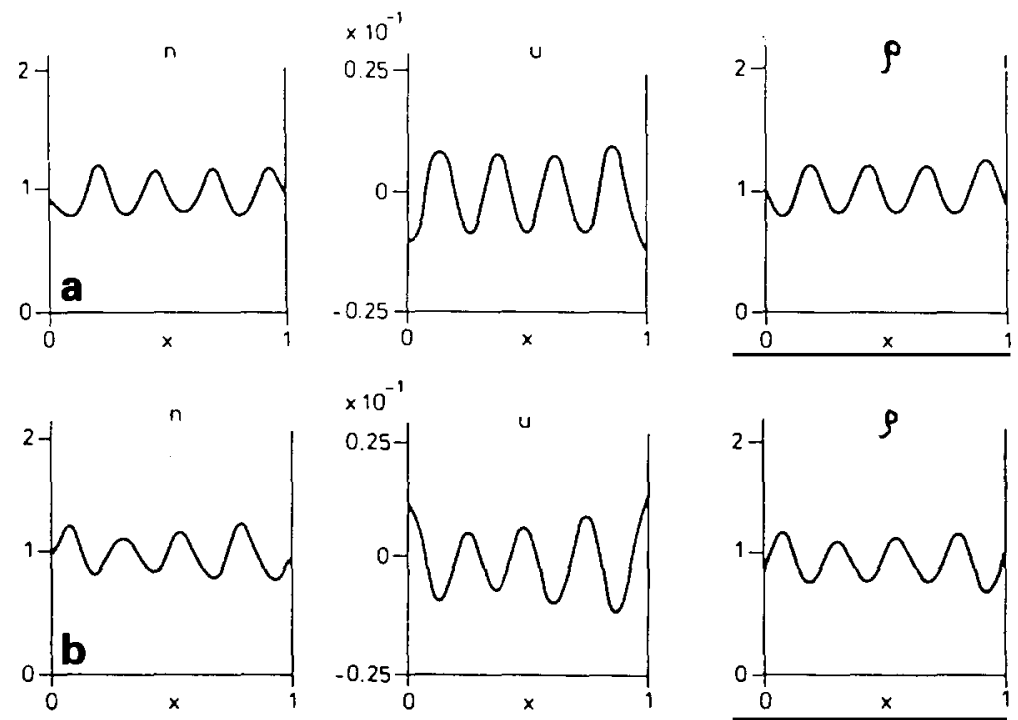

Fig. 4.2. Steady state solutions, for the cell density $n$, ECM density $\rho$ and ECM displacement $u$, of the nonlinear one-dimensional mechanical model $(28-30)$. (a) Periodic boundary conditions were used and initial conditions were random perturbations about the uniform steady state $n=\rho=1$, $u=0$. (b) Heterogeneous steady state solutions with the initial stress pattern in (a). Parameter values: $D_{1}=a=\gamma=10^{-3}, \lambda=0.12, \tau=1.65, s=400$, $\mu=1$. Note that $n$ and $\rho$ are in phase and both out of phase with $u$. 
and epidermis. It is well known that mechanical deformations affect mitosis and so tissue interaction is a natural effect to include in mechanical models: see also the discussion in Oster et al. [1983]. Nagorcka et al. [1987] investigated a tissue interaction mechanism specifically with the complex patterns of scales in mind.

\section{Cartilage condensations in limb morphogenesis}

The vertebrate limb is one of the most widely and easily studied developmental systems and such studies have played a major role in embryology. Experimental evidence from amphibians suggests that osmotic properties of the ECM may be important in morphogenesis. Hyaluronate is a principal component of the ECM and can exist in a swollen osmotic state. As the condensation of chondrocytes starts the cells secrete an enzyme, hyaluronidase, which degrades the hyaluronate. This could lead to the osmotic collapse of the matrix thus bringing the cells into close enough contact to initiate active contractions and thus generate cell aggregations. Cell motility is probably not important in this scenario.

A mechanical model for generating the pattern of cell condensations which evolve in a developing limb bud and which eventually becomes cartilage was first put forward by Murray et al. [1983] and Oster et al. [1983]. A modification of the mechanical model to incorporate the above chemical aspects and the added forces caused by osmotic pressure has been proposed and analysed by Oster et al. [1985]. They showed that such a mechanochemical model would generate similar chondrogenic patterning for the developing limb to that obtained with the earlier mechanical model.

The pattern in developing limb buds which determines the final cartilage patterns, which later ossify into bones, involves aggregations of chondrocyte cells, which are mesenchymal cells such as we have been considering. The basic evolution of chondrocyte patterns takes place sequentially as the limb bud grows, which it does from the distal end. Figure 5.1 gives an explanation of how, with geometry and scale as bifurcation parameters, chondrogenesis could proceed. The actual sequence of patterns for the developing chick limb is illustrated in fig. 5.1c. The detailed explanation of the process based on a mechanical mechanism is the following.

As the limb bud grows, through cell proliferation at the distal end, the cross section of the tissue domain, which includes the ECM and mesenchymal cells, is approximately circular but with an elliptical bias. Let us consider this to be the two-dimensional domain for our mechanical model with zero flux boundary conditions for the cells $n$ and matrix $\rho$. The condition for $u$ is an imposed restraining force which comes from the epidermis - the sleeve of the limb bud. We suppose that as the cells age the traction increases and eventually passes through the critical values $\tau_{c}$. The detailed form of the dispersion relation is such that the first bifurcation produces a single central aggregation of cells recruited from the surrounding tissue.

The axial cell aggregations are influenced by the cross-sectional shape as shown in fig. 5.1a. As the cells condense into a single aggregation they generate a strong centrally directed stress as in fig. 5.1b. This radial stress deforms the already slightly elliptical cross section to make it even more elliptical which in turn induces a bifurcation to two condensations because of the changed flatter geometry of the cross section. An aerofoil section gives rise to two condensations of different size.

As distinct from chemical prepattern models mechanical models influence the shape of the domain and can actually induce the sequence of bifurcations shown in fig. 5.1c. 


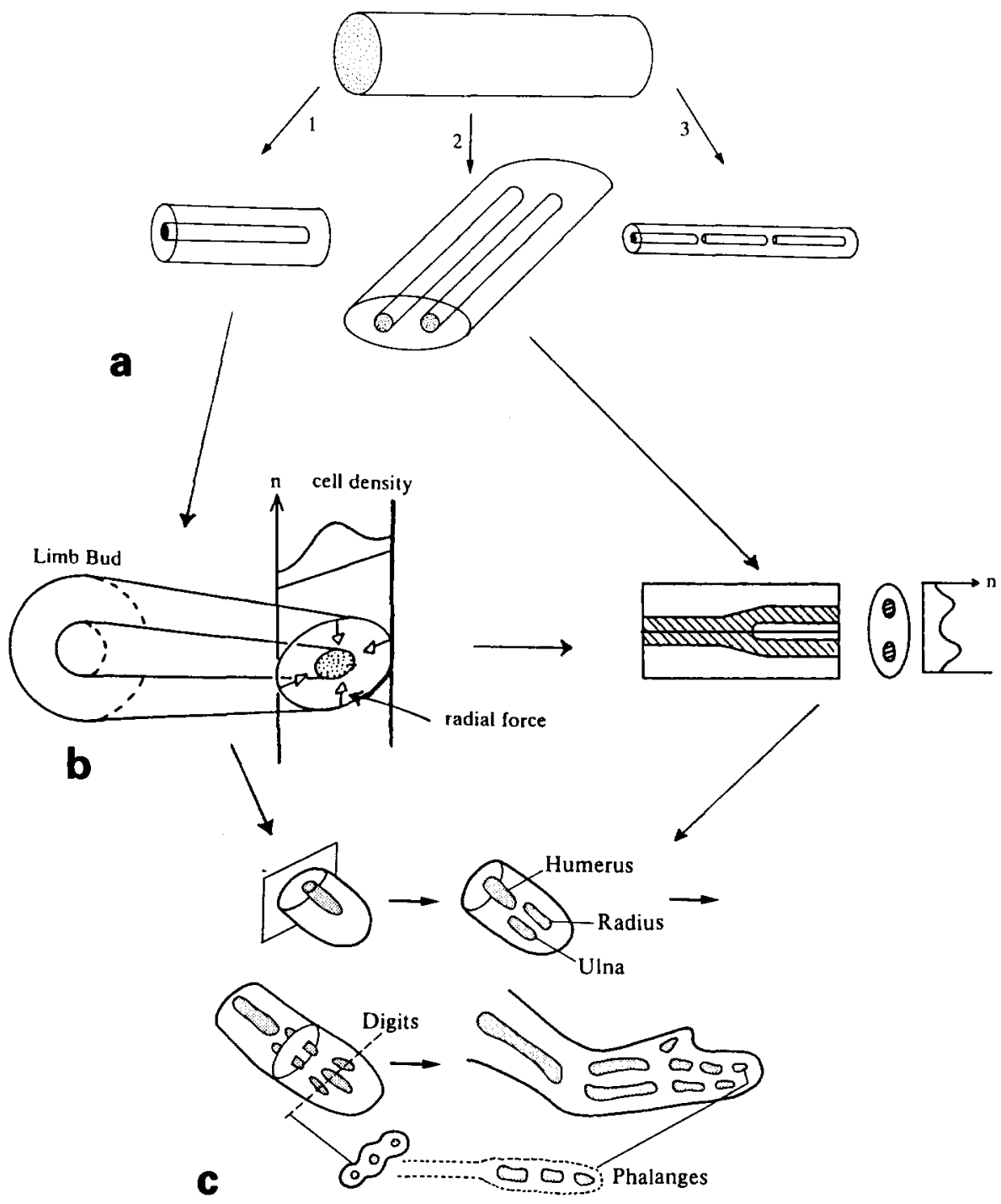

Fig. 5.1. (a) The type of axial condensation is influenced by the cross-sectional shape of the limb. Initially a single condensation, path 1 , will be produced (for example, the humerus in (c)). A more elliptical cross section allows two aggregations to form, path 2. An aerofoil-shaped domain produced unequal condensations (for example, the radius and ulna in (c)). In a long thin cylinder the axial condensations form segmental units, path 3 (for example, the phalanges in (c)). (b) This shows how the mechanical mechanism influences cross-sectional form and hence induces the required sequence of chondrogenic patterns. As the cells form the central condensation their tractions deform the limb to be more elliptical. At a critical ellipticity the pattern bifurcates to two condensations. How three condensations are formed is important and explained in the text: refer also to fig. $5.2 \mathrm{~d}$. (c) The schematic bifurcation sequence of chondrocyte (mesenchymal) cell aggregations which presage cartilage formation in the developing chick limb.

After a two-condensation state has been obtained, further growth and flattening can generate the more distal patterns. By the time the limb bud is sufficiently flat, cell traction recruitment effectively isolates patterning of the digits. The domain can now be considered linear and subsequent growth induces longitudinal or segmental bifurcations with more condensations simply fitted in as the domain increases and we get the simple laying down of segments, for example, the phalanges in fig. 5.1c, as predicted by fig. 5.1a, path 3 . 
The sequence of cell pattern bifurcations need not be generated by a changing geometry; it can result from a variation of other parameters in the model. Also asymmetric condensations can result from a spatial variation or asymmetry in a parameter across the limb cross-section. There is well-documented experimental evidence for asymmetric properties, which, of course, are reflected in the different bone shapes and sizes in the limb such as the radius and ulna in fig. 5.1c. Whatever triggers the bifurcations as we move from the proximal to distal part of the limb the natural sequence is from a single condensation to two condensations and then to several.

\section{Morphogenetic rules for cartilage morphogenesis in the limb}

With a completely symmetric geometry and tissue isotropy it is possible to move through the bifurcation space of parameters from one aggregation to two to three and so on. With the natural anisotropy in embryological tissue such isotropy does not exist. The question then arises as to how the pattern sequence from a double to a triple condensation is effected. During chondrogenesis there appears to be little cell division, which implies that condensations principally form through recruitment of cells from neighbouring tissue. Thus, as the limb bud grows the pattern bifurcation that takes place following a branching bifurcation is as illustrated in fig. 5.2c. Figures 5.2a, b show the other two basic condensation elements in setting up a cell condensation pattern in a developing limb. We believe that for all practical purposes the process giving rise to triple bifurcation must be that in which one branch of the double condensation itself undergoes a branching bifurcation.

If we now take the bifurcating pattern elements in figs. $5.2 \mathrm{a}-\mathrm{c}$ as the three allowable types of cell condensations we can see how they can be used to construct any limb cartilage pattern. As an example, fig. 5.2e illustrates this for the forelimb of a salamander. So, even without considering any specific mechanism, we hypothesise an important set of morphogenetic rules for the patterning sequence of cartilage in the development of the vertebrate limb. This hypothesis, encapsulated in the theory put forward by Murray et al. [1983] and Oster et al. [1983], has recently been exploited by Oster et al. [1988] who present extensive experimental evidence for its validity. They also discuss aspects of the theory and its predictions which are of considerable potential importance in evolutionary biology.

We believe these rules are model independent, or rather any model mechanism for chondrogenic pattern formation must be capable of generating such a sequence of bifurcating patterns.

A major role of theory in morphogenesis is to suggest possible experiments to distinguish between different models each of which can generate the appropriate sequence of patterns observed in limb chondrogenesis. Mechanical models lend themselves to experimental scrutiny more readily than reaction diffusion models because of the elusiveness of chemical morphogens.

\section{A mechanochemical model for wound healing}

The process of cutaneous wound healing often involves some degree of wound contraction, the movement of the wound boundary and adjacent uninjured tissue towards the wound centre. In some cases, contraction can be advantageous for wound closure; however, it can create functional impairment (e.g. when contraction occurs near a joint) as well as be cosmetically unattractive (e.g. facial wounds) in others. Experimental investigations have demonstrated that fibroblast cells repopulate a wound and are responsible for contraction [Rudolph 1979]. Thus, cutaneous wound contraction is clearly a physiological process in which cell forces are of great importance. The mechanochemical modelling approach not only provides a framework for understanding how events in wound healing are orchestrated so as to 


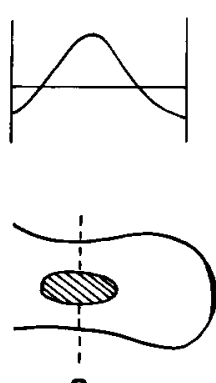

a
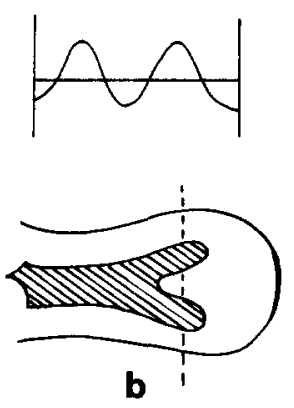

b
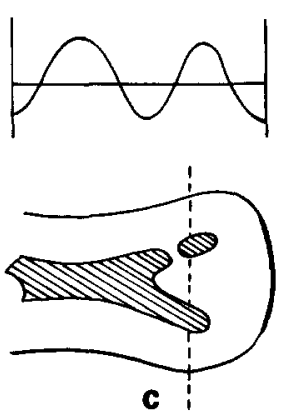
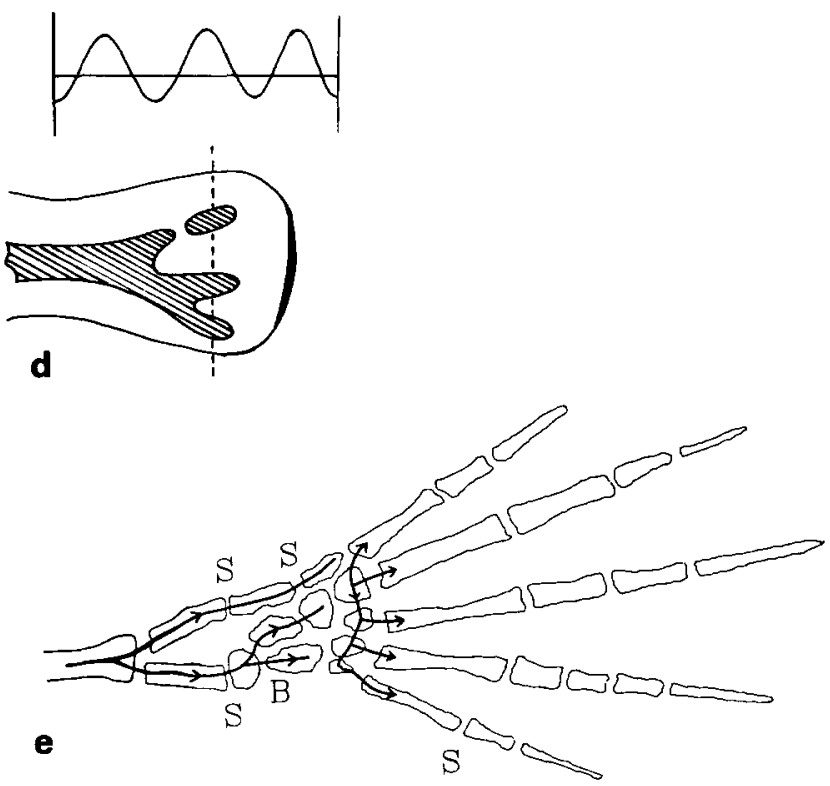

Fig. 5.2. (a-c) The three basic types of cell condensations which generate cartilage patterns in the developing vertebrate limb. These are postulated as the morphogenetic rules for cartilage pattern generation for all vertebrate limbs. (a) Focal condensation F; (b) Branching bifurcation B; (c) Segmentation condensation $S$. (d) Formation of more patterns is by further branching or independent foci. (e) An example of a branching sequence showing how the cartilage patterns in the limb of a salamander can be built up from a sequence of $F, B$ and $S$ bifurcations.

give rise to contraction, but also a predictive means for enhancing or mitigating contraction, as is appropriate for a particular wound. There is also considerable evidence that cutaneous wound healing can serve as a model for understanding a whole class of soft tissue pathologies, from fibrotic disorders to generation of tumours [Seemayer et al. 1981].

Wound contraction occurs in the context of other phases of wound healing which may be influential in the initiation and regulation of contraction [Clark 1985]. Inflammatory cells are believed to release a host of chemical stimuli for fibroblasts (e.g. growth factors and chemoattractants) that govern the subsequent phases of repair: biosynthesis of wound matrix followed by an extended period of matrix remodelling, which typically results in a dense collagenous scar. Contraction usually parallels the biosynthetic phase when collagen and other matrix macromolecules replace the initial fibrin clot. The close temporal relationship of contraction with inflammation and matrix biosynthesis provides two scenarios to be considered within the basic mechanochemical model. 
Unlike for the previous applications of the model, there are quantitative data already available for cutaneous wound contraction for comparison with model predictions. In a study by McGrath and Simon [1983] addressing the longstanding controversy of what type of wound closes fastest, they observed that the contracting phase of "full thickness" dermal wounds on rats could be described with a simple exponential dependence on time:

$$
A=A_{\mathrm{f}}+\left(A_{0}-A_{\mathrm{f}}\right) \mathrm{e}^{-k t}
$$

where $A_{0}$ is the wound area when contraction begins and $A_{\mathrm{f}}$ is the area remaining after contraction is completed (areas are scaled to the excised area). The time constant, $k$, was found to be independent of both the initial wound area and wound geometry for the limited cases studied $\left(6.25 \mathrm{~cm}^{2}\right.$ and $12.54 \mathrm{~cm}^{2}$ squares and $12.54 \mathrm{~cm}^{2}$ circles).

In addition to assessing the model's admissability of such quantitative predictions, it is necessary to consider what qualitative features relevant to wound contraction should be intrinsic to the model. A rather obvious one serves to constrain the permissible parameter space: unwounded skin should be in some sense stable to at least small perturbations. Given the pattern forming capabilities of the models described above, this is not a trivial consideration. This is addressed using linear stability analysis of the uniform state of the type outlined earlier. Other relevant features are not as objective, but reflect the typical outcome of a wound: a rapid initial expansion of the wound followed by a longer period of contraction with increase in collagen content.

The base model upon which the two scenarios are considered assumes that the cells move by random dispersion and convection only and proliferate according to a logistic rate law. (Other modes of fibroblast migration such as chemotaxis and contact guidance, although not considered here, are generally believed to play a significant role in fibroblast repopulation of a wound space. Also there are many speculated effects of biochemical mediators on fibroblast behaviour, ranging from oxygen concentration modulation of proliferation to inflammation-derived growth factor-modulation of traction. The latter, a key element is considered in the first scenario here.) The dermis is again assumed to be a matrix adequately described by a linear, isotropic, viscoelastic stress tensor modified with the local cell tractional force term. (Thus osmotic forces which develop in the wound space are neglected here, as are any consequences of matrix fibre anisotropy which might develop during wound healing.) The attachment of the dermis to underlying anatomical features is modelled by the linearly elastic body force. Virtually all animal models used for the study of wound healing possess the panniculus carnosus, a skin organ underlying the dermis which is absent in humans and confers great mobility to animal dermis relative to human dermis in response to stress. This may be accounted for at the simplest level by considering the elastic constant associated with underlying attachment of the skin, $s$, to be small and large respectively. The system of equations for this base model takes the form:

$$
\begin{aligned}
& n_{t}+\nabla \cdot\left(n u_{t}\right)=D_{1} \nabla^{2} n+r n(1-n), \\
& \nabla \cdot\left\{\mu_{1} \varepsilon_{t}+\mu_{2} \theta_{t} I+E\left(\varepsilon+\nu^{\prime} \theta I\right)+\tau n \rho l /\left(1+\lambda n^{2}\right)\right\}=s \rho u, \\
& \rho_{t}+\nabla \cdot\left(\rho u_{t}\right)=0 .
\end{aligned}
$$

The associated dimensionless variables and parameters are the same as in eq. (17).

The idealization of the initial state of a wound is that $u=0$ and $\rho=1$ for all $x$ in the (infinite) 
domain, and that inside some initial wound boundary, $x_{\mathrm{b}}, n=0$ while for all $x$ outside the boundary, $n=1$. This implies that the wound matrix (that is, fibrin clot) is formed instantaneously relative to the contraction phase and that it is devoid of fibroblasts, which are accurate assumptions [Clark 1985]. Further, it is assumed that the mechanical properties of the wound matrix are the same as the surrounding dermis, and this remains true for the wound matrix for all times as it becomes modified due to cell biosynthesis. These are much less accurate assumptions but appropriate given the complex modifications of the wound matrix composition that occurs during wound healing [Clark 1985, Weigel et al. 1986].

Although it can be shown that this base model admits the occurrence of a stable nonuniform steady-state evolving from the initial state, neither the qualitative features of such a steady-state nor its evolution from the initial state are consistent with those of a contracting wound. These deficiencies are remedied in both of the following scenarios.

In the first scenario, we assume for simplicity that the inflammatory mediator exists as a stable concentration gradient exponentially decreasing from the wound centre over the time-course of contraction (models of inflammation provide a basis for extending this simple description [see Alt and Lauffenburger 1987]). There are various mechanisms by which this mediator could influence the behavioural properties of fibroblasts. Experimental evidence indicates that fibroblasts transform into highly contractile myofibroblasts near a wound due to some mediator [Skalli and Gabianni 1987] and suggests that the traction parameter, $\tau$, should depend on mediator concentration, $c$, according to

$$
\tau=\tau_{0}\left\{1+\tau_{\mathrm{f}} c /(1+c)\right\}
$$

where $\tau_{\mathrm{f}}$ is a traction enhancement coefficient. $c$ is taken to have the following dependence on distance $x$ from the wound centre,

$$
c=c_{0} \exp \left[-x^{2} / \sigma\right]
$$

where $c_{0}$ is the concentration at the wound centre scaled to the concentration for half-maximal traction enhancement and $\sigma$ is a parameter specifying the spatial domain of influence of the mediator from the wound centre.

Assuming matrix strains are sufficiently small we can linearise the conservation equation for $\rho$ in eq. (33): in one space dimension

$$
\rho_{t}+\left(u_{t}\right)_{x}=0 \Rightarrow \rho=1-u_{x}
$$

If we further assume the cell density in the steady state is essentially $n=n_{\mathrm{s}}=1$, which is a solution of eq. (31), an approximation to the steady-state profile $u_{\mathrm{s}}(x)$ is given by substituting for $\rho$ from eq. (34) into eq. (32) to get

$$
\left(1-\frac{\underline{\tau}}{1+\lambda}\right) \frac{\mathrm{d}^{2} u_{\mathrm{s}}}{\mathrm{d} x^{2}}=\left(1-\frac{\mathrm{d} u_{\mathrm{s}}}{\mathrm{d} x}\right)\left(s u_{\mathrm{s}}-\frac{\mathrm{d} \tau / \mathrm{d} x}{1+\lambda}\right) .
$$

The solution of eq. (35) gives the final contracted state of the wound matrix displacement, $u_{\mathrm{s}}$, given the persistent gradient of inflammatory mediator. The profiles obtained in this manner agree well with those obtained from numerical solution of the full system of equations (31-33). An example of the transient profiles is given in fig. 6.1 for the case of linear wound geometry. 

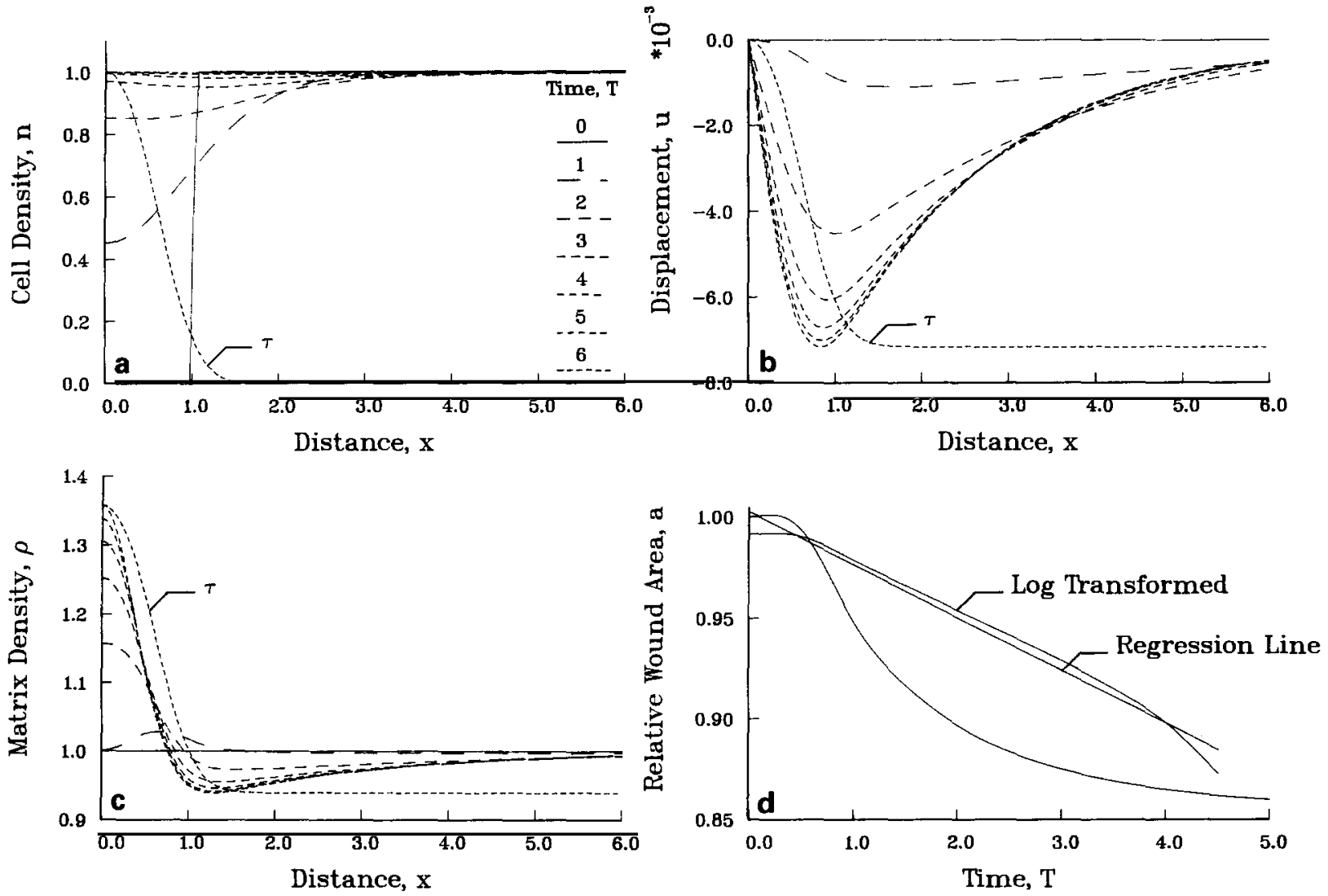

Fig. 6.1. Numerical results for the variable traction case of the wound contraction model for linear geometry: (a) cell density, $n$; (b) displacement of cell/matrix composite, $u ;$ (c) and matrix density, $\rho$, are plotted versus distance, $x$, from the wound centre for a series of times after wounding. The imposed traction gradient is indicated by the dashed line $\left(\tau_{\mathrm{f}}=2.5, c_{0}=1\right)$. In (d) the relative wound area, $a$, is plotted as a function of time ( $a>1$ is associated with wound expansion, $a<1$ is associated with wound contraction). The straight line is the linear least squares regression line of $a$ transformed according to the exponential form suggested by McGraw and Simon [1983]. See text for further discussion. Parameter values: $D_{1}=0.001, \mu=1, r=1, s=100, \tau_{0}=0.5, \lambda=1.5, \sigma=0.4$, where $\mu=\mu_{1}+\mu_{2}$, and $\mu, s$ and $\tau_{0}$ are all scaled by $1+\nu^{\prime}$.

The repopulation of the wound space by cells (that is fibroblasts) by random migration and proliferation is seen in fig. 6.1a to restore the cell concentration to that characteristic of the unwounded dermis. The evolution of the matrix to a contracted steady-state is apparent in fig. 6.1b. Associated with the contraction is an increase in the matrix density as seen in fig. 6.1c. The plot of the wound area over time relative to the initial wound area in fig. 6.1d, which is identical to a plot of the "contracting phase" as analysed by McGrath and Simon, suggests that the cell-matrix-mediator mechanisms of this scenario can conspire to yield the empirical exponential description which they observed. The time constant, $k$, calculated from the numerical simulation is independent of the initial wound area, in agreement with their findings, since the solutions are obtained for dimensionless variables on an infinite domain. We found that the analogous simulation in cylindrical polar coordinates gives approximately the same value for $k$ (1.00 versus 1.01), which is also in agreement with McGrath and Simon's [1983] related finding that $k$ is independent of geometry. 
In the second scenario, the basic model is modified to include a matrix biosynthesis term, accounting for the extensive deposition of collagen and other matrix macromolecules by fibroblasts in the wound region. Since the functional dependence of this biosynthesis rate on $n, u$ and $\rho$ is not yet known, we assume for illustrative purposes here that net rate of biosynthesis is proportional to that of proliferation (that is, biosynthesis has a logistic-type rate law, $b n(1-n)$ ). A priori prediction of a contracted steady-state is more difficult with this model scenario. An example of the numerical solution of eqs. (31-33) with the biosynthesis term included in eq. (33) is shown in fig. 6.2, again for a linear wound. It shares many similarities with the other scenario, including a contracted steady-state reasonably described with an exponential which is again independent of initial wound area and approximately the same for both linear and circular wound geometries (1.02 versus 1.03$)$.

There are many scenarios which can be considered given the complexity of the wound healing phenomenon. The mechanochemical model offers a possibility of considering the effects of various known and speculated responses and dermal/wound matrix properties individually as well as collectively. An improved understanding of how these effects are interrelated could lead to new possibilities for controlling wound contraction and optimizing the wound healing response. Such initial success is encouraging for future work in this area and such studies are currently in progress.
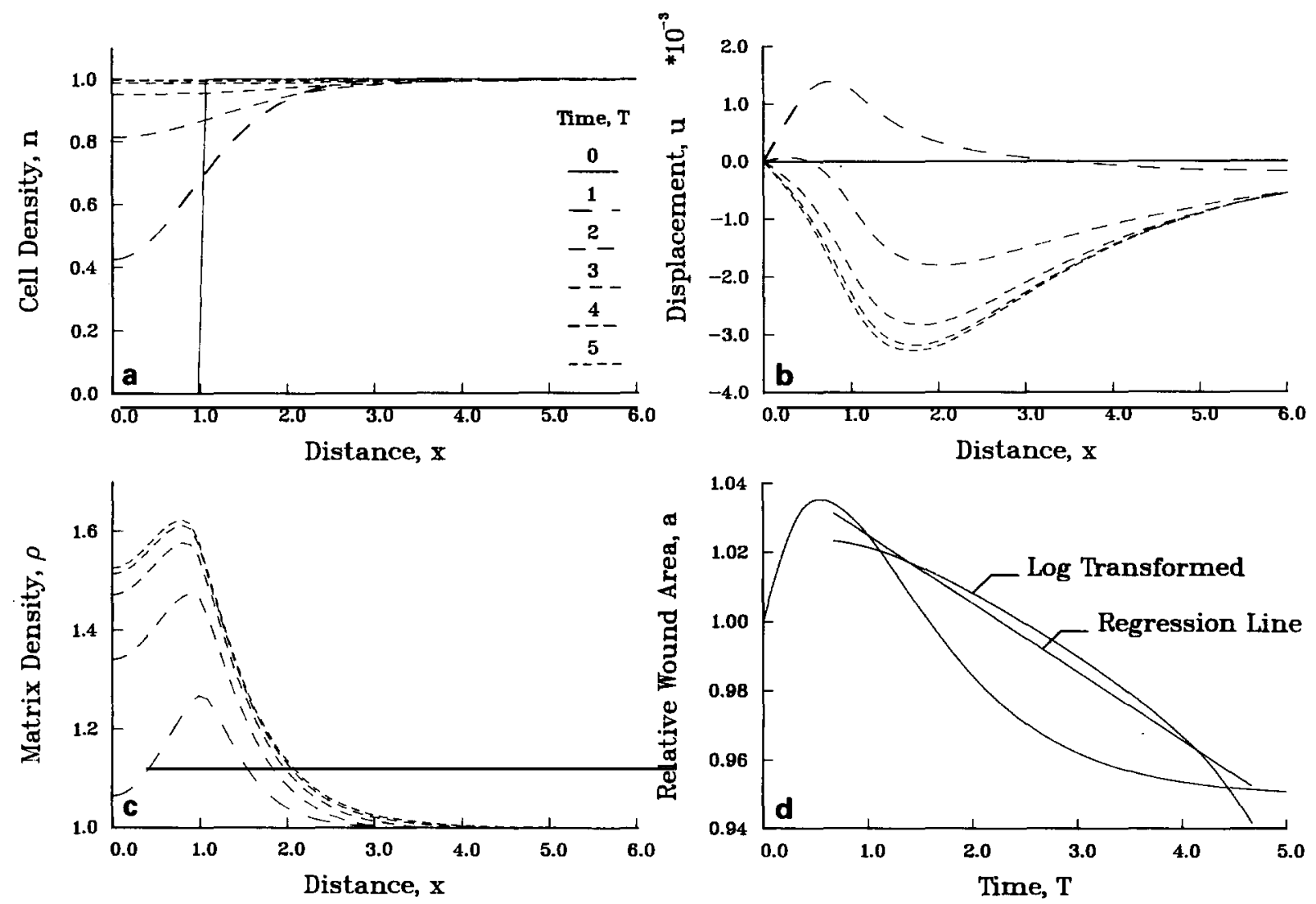

Fig. 6.2. (a-d) Numerical results for the matrix biosynthesis case of the wound contraction model for linear geometry. The parameter values are the same as those used in fig. 6.1 where a general description of this plot is given, except that $\tau_{\mathrm{f}}=0$ and $b=1$. See the text for further discussion. 


\section{Other applications of mechanochemical models}

There are many potential applications which have not yet been examined in depth or even not at all. Here we mention a few, but only very briefly.

(i) Postfertilization waves on eggs. These waves are associated with pairs of mechanical and chemical phenomena observed on the surface of many vertebrate eggs shortly after fertilization. The waves effectively emanate from the sperm entry point and sweep over the egg. A mechanical model for the egg surface based on a contractile machinery, similar to that involved in muscle contraction, wherein the contraction is intimately related to the calcium chemistry involved has been developed and studied by Cheer et al. [1987] and Lane et al. [1987]. In the latter paper analytical results are obtained for the wave profiles of the mechanical and chemical waves and the results compare well qualitatively with observations.

(ii) Animal coat patterns. Murray (1981) showed that many of the patterns observed on mammalian coat patterns could be generated with a morphogen based reaction diffusion mechanism. The patterns thus generated were considered the chemical prepatterns to which the melanoblast cells, the precursors of melanocytes, the pigment forming cells respond. The evidence presented for such a theory was based on observational comparisons and on certain developmental constraints which are dictated by the geometry and scale of the animal's surface when the pre-pattern is laid down. These melanoblast cells migrate from the neural crest early in development. Since the model discussed in section 2 deals specifically with such migratory cells it is possibly directly applicable to patterns found on mammalian coats. In view of the evident richness of patterns which our mechanical models can generate, it is clear that we can obtain not only similar patterns to those from a reaction diffusion mechanism but others which the latter cannot exhibit.

(iii) Rejection of artificial joints. One of the problems with artificial hip joints is that the cement for fixing them inside the femur does not form a good bond with the living tissue. The concepts used in setting up the cell motility mechanisms suggest that a way to effect a more suitable bond might be to try and produce an adhesive which is sufficiently porous to allow movement of cells into it, by virtue of the cell tractions.

\section{Discussion}

There can be no doubt that mechanochemical processes are involved in development. The models we have described here represent a new approach and the concepts suggest that mechanical forces could be the principal guiding elements in producing the correct sequence of tissue patterning and shapes which are found in the developing embryo. Whereas in reaction diffusion models a chemical morphogen prepattern is set up, which is then read and interpreted by the cell as required, in a mechanochemical framework pattern formation and morphogenesis are one and the same.

The models simply reflect the laws of mechanics as applied to tissue cells and their environment and are based on known biological and biochemical facts: all of the parameters involved are in principle measurable.

We should add that these models are first attempts, and considerable mathematical analysis is required to investigate their potentialities to the full. In turn this will suggest model modifications in the usual way of realistic biological modeling. At this stage the analysis has only just been started but is sufficient to indicate a wealth of wide-ranging patterns and mathematically challenging problems. The 
models have already been applied realistically to a variety of morphogenetic problems of current major interest. The results and basic ideas have initiated considerable experimental investigation and new ways of looking at a wide spectrum of embryological problems.

The emergence of spatial pattern in the embryo is still unresolved. No mathematical model can take into account the very complex physical, chemical and electrical processes involved in morphogenesis. Every mathematical model is a gross simplification of the actual processes involved. However, in making such simplifications, one is trying to capture the essential mechanisms involved. The role of mathematical models is to try to highlight the possible interactions between specific biological processes and to suggest relevant experiments. They have generated and directed a great deal of experimental activity among biologists, and from this point of view, such models for morphogenesis are fulfilling their role.

\section{Acknowledgements}

PKM would like to acknowledge the Science and Engineering Research Council of Great Britain for its support (Grant GR/c/63595). RTT was supported by a NATO postdoctoral fellowship. RTT would also like to thank the Centre for Mathematical Biology for its hospitality during the tenure of his NATO fellowship.

\section{References}

Alt, W. and D.A. Lauffenburger, 1987, Transient behaviour of a chemotaxis system modelling certain types of tissue inflammation, J. Math. Biol. 24, 691-722.

Britton, N.F., 1986, Reaction-Diffusion Equations and their Applications to Biology (Academic Press, New York).

Cheer, A., R. Nuccitelli, G.F. Oster and J.-P. Vincent, 1987, Cortical activity in vertebrate eggs I: The activation waves, J. Theor. Biol. 124, 377-404.

Clark, R.A.F. 1985, Cutaneous tissue repair: Basic biological considerations, J. Am. Acad. Dermatol. 13, 701-725.

Cohen, D.S. and J.D. Murray, 1981, A generalized diffusion model for growth and dispersal, J. Math. Biol. 12, $237-249$.

Davidson, D., 1981, The mechanism of feather pattern development in the chick. I. The time of determination of feather position. II. Control of the sequence of pattern formation, J. Embryol. Exp. Morph. 74, 245-273.

Davidson, D., 1983a, The mechanism of feather pattern development in the chick. I. The time determination of feather position, J. Embryol. Exp. Morphol. 74, 245-259.

Davidson D., 1983b, The mechanism of feather pattern development in the chick. II. Control of the sequence of pattern formation, J. Embryol. Exp. Morphol. 74, 261-273.

Dhouailly, D., 1975, Formation of cutaneous appendages in dermo-epidermal recombinations between reptiles, birds, and mammals, Wilhelm Roux's Arch. Devl. Biol. 177, 323-340.

Field, R.J. and M. Burger, eds, 1985, Oscillations and Travelling Waves in Chemical Systems (Wiley, New York).

Fife, P.C., 1979, Mathematical Aspects of Reacting and Diffusing Systems. Lect. Notes in Biomathematics 28 (Springer, Berlin).

Harris, A.K., D. Stopak and P. Wild, 1981, Fibroblast traction as a mechanism for collagen morphogenesis, Nature 290, $249-251$.

Landau, L. and E. Lifshitz, 1970, Theory of Elasticity (2nd edition) (Pergamon, New York).

Lane, D.C., J.D. Murray and V.S. Manoranjan, 1987, Analysis of wave phenomena in a morphogenetic mechanochemical model and an application to post-fertilisation waves on eggs, IMA J. Math. Applied in Medic. and Biol. 4, 309-331.

Lauffenburger, D.A. and W. Alt, 1987, Transient behavior of a chemotaxis system modelling certain types of tissue inflammation, J. Math. Biol. 24, 691-722.

Maini, P.K. and J.D. Murray, 1988, A nonlinear analysis of a mechanical model for biological pattern formation, SIAM J. Appl. Math. (in press). McGrath, M.H. and R.H. Simon, 1983, Wound geometry and the kinetics of contraction, Plast. Reconstr. Surg. 72, 66-72.

Meinhardt, H., 1982, Models of Biological Pattern Formation (Academic Press, London).

Murray, J.D., 1977, Nonlinear Differential Equation Models in Biology (Clarendon Press, Oxford). 
Murray, J.D., 1981, On pattern formation mechanisms for lepidopteran wing patterns and mammalian coat markings, Philos. Trans. R. Soc. London B 295, 473-496.

Murray, J.D., 1988, Mathematical Biology (Springer, Berlin) (in press)

Murray, J.D. and G.F. Oster, 1984a, Generation of biological pattern and form, IMA J. Math. Appl. in Medic. and Biol. 1, 51-75.

Murray, J.D. and G.F. Oster, 1984b, Cell traction models for generating pattern and form in morphogenesis, J. Math. Biol. $19,265-279$.

Murray, J.D., G.F. Oster and A.K. Harris, 1983, A mechanical model for mesenchymal morphogenesis, J. Math. Biol. $17,125-129$.

Nagorcka, B.N., V.S. Manoranjan and J.D. Murray, 1987, Complex spatial patterns from tissue interactions - an illustrative model, J. Theor. Biol. $128,359-374$.

Oster, G.F., J.D. Murray, and A.K. Harris, 1983, Mechanical aspects of mesenchymal morphogenesis, J. Embryol. Exp. Morphol. 78, 83-125.

Oster, G.F., J.D. Murray and P.K. Maini, 1985, A model for chondrogenic condensations in the developing limb: the role of extracellular matrix and cell tractions, J. Embryol. Exp. Morphol. 89, 93-112.

Oster, G.F., N. Shubin, J.D. Murray and P. Alberch, 1988, Evolution and morphogenetic rules. The shape of the vertebrate limb in ontogeny and phylogeny, Evolution (in press).

Penrose, R., 1979, The topology of ridge systems, Ann. Hum. Genet. London 42, 435-444.

Rawles, M., 1963, Tissue interactions in scale and feather development as studied in dermal-epidermal recombinations, J. Embryol. Exp. Morph. $11,765-789$

Rudolph, R., 1979, Location of the force of wound contraction, Surg. Gynecol. Obstet. 148, 547-551.

Seemayer, T.A., W. Schurch and R. Lagace, 1981, Myofibroblasts in human pathology, Hum. Path. 12, 491-492.

Sengel, P., 1976, Morphogenesis of Skin (Cambridge University Press, Cambridge).

Skalli, O. and G. Gabbiani, 1988, The biology of the myofibroblast in relation to wound contraction and fibrocontractive diseases, in: The Molecular and Cellular Biology of Wound Repair, eds R.A.F. Clark and P.M. Henson (Plenum, New York) pp. 373-388.

Smoller, J., 1983, Shock Waves and Reaction-Diffusion Equations (Springer, Berlin).

Turing, A.M., 1952, The chemical basis of morphogenesis, Phil. Trans. R. Soc. London B 237, 37-72.

Weigel, P.H., G.M. Fuller, and R.D. LeBoeuf, 1986, A model for the role of hyaluronic acid and fibrin in the early events during inflammatory response and wound healing, J. Theor. Biol. 119, 219-234.

Wessells, N., 1977, Tissue Interaction in Development (W.A. Benjamin, Menlo Park).

Wolpert, L., 1969, Positional information and the spatial pattern of cellular differentiation, J. Theor. Biol. 25, 1-47.

Wolpert, L., 1971, Positional information and pattern formation, Curr. Top. Dev. Biol. 6, 183-224. 